\title{
Relationship of Prostate-Specific Antigen Level With Obesity Indices in Korean Middle-Aged Population
}

\section{Seung Ki Min, Kwibok Choi, Byoung Hoon Kim, In-Chang Cho}

Department of Urology, National Police Hospital, Seoul, Korea

\begin{abstract}
Purpose: We evaluated the relationship of prostate-specific antigen (PSA) and obesity indices (weight, body mass index [BMI] and waist circumference [WC]) in Korean middle-aged men.

Materials and Methods: From February to September 2013, 1,900 police men under 60 years old who participated in a prostate health screening program were included this cross-sectional study. All subjects underwent clinical examinations including weight, height, BMI, WC, fasting blood sugar, lipid profiles, estimated glomerular filtration rate (GFR), and PSA. Total prostate volume (TPV) was assessed clinically. Spearman correlation and multiple linear regression tests were performed to evaluate the obesity indices and PSA relationships.

Results: The mean age was $52.0 \pm 4.7$ years, and the mean PSA was $0.97 \pm 0.99 \mathrm{ng} / \mathrm{mL}$. The PSA showed a significant positive correlation with the age $(r=0.108, p<0.01)$, TPV $(r=0.349, p<0.01)$, height $(r=-0.052, p<0.05)$, weight $(r=0.186, p<0.05)$, low-density lipoprotein cholesterol $(r=0.056, p<0.05)$, and $G F R(r=-0.096, p<0.01)$. All obesity indices including weight, BMl, and WC showed negative correlations with PSA (beta=-0.013, $p<0.001$; beta $=-0.039, p<0.001$; and beta $=-0.010, p=0.005$; respectively) in age and TPV-adjusted model.

Conclusions: Common obesity indices (weight, BMI, and WC) were associated with lower PSA in Korean middle-aged population. Thus, an individual's degree of obesity should be considered when PSA is checked in the first prostate cancer screening of life. (Korean J Urol Oncol 2018;16:103-109)
\end{abstract}

Key Words: Prostate-specific antigen $\cdot$ Middle-age $\cdot$ Obesity $\cdot$ Prostate cancer $\cdot$ Screening

\section{INTRODUCTION}

Prostate cancer $(\mathrm{PCa})$ is a major cause of death and the second most frequently diagnosed malignancy among men in the

Received September 8, 2018, Revised September 29, 2018,

Accepted October 10, 2018

Corresponding Author: In-Chang Cho

Department of Urology, National Police Hospital, 123 Songi-ro, Songpa-gu, Seoul 05715, Korea

E-mail: uroic@outlook.kr

Tel: +82-2-3400-1264, Fax: +82-2-431-3192

ORCID code: https://orcid.org/0000-0001-8906-3478

- This study was supported by the National Police Hospital Grant of clinical research. world, accounting for about $15 \%$ of total cancer diagnoses. ${ }^{1}$ In South Korea, PCa incidence has increased steadily, while its mortality rate has declined slowly, due to extension of life expectancy of Korean, trend of western dietary style, and increased PSA screening. ${ }^{1}$

To date, the proven risk factors for PCa are age, race, family history, and genetic factors. The rapid economic growth of developing countries and the widespread western diet have changed the pattern of disease in Korean over the past decade. The increase in obesity and metabolic syndrome in Korean population is a typical change. It was reported that obesity is strongly associated with the development of advanced PCa in a recent meta-analysis, indicating that acquired risk factors re-

(i) (3) This is an Open Access article distributed under the terms of the Creative Commons Attribution Non-Commercial License (http://creativecommons.org/licenses/by-nc/4.0/) which permits unrestricted non-commercial use, distribution, and reproduction in any medium, provided the original work is properly cited. 2018 (C) Copyright The Korean Urological Oncology Society and The Korean Prostate Society. All Rights Reserved. 
lated to life style are associated with aggressiveness and progression of PCa. In the study of United States, Gleason score 7 or more PCa had positive correlations with body mass index (BMI), waist circumference (WC), body fat mass and fat-free mass. $^{2}$ In addition, in large European cohort study, abdominal fat had been shown to increase the risk of advanced $\mathrm{PCa}^{2} \mathrm{~A}$ prospective cohort study in Australia revealed that weight, BMI and weight gain in adulthood were associated with the development of high risk PCa, but not with the all PCa risk. ${ }^{3}$ In addition, recent some studies on Asian populations have shown that PSA is age-dependent, increasing gradually with age, but negatively correlated with BMI. It is usually thought to be due to hemodilution by increasing body fluid volume. ${ }^{4}$

In addition to obesity, other components of metabolic syndrome are also known to be associated with the risk of PCa. However, the relationship between lifestyle-related risk factors, such as dyslipidemia and diabetes, and the development and progression of $\mathrm{PCa}$ remains unclear and has not been concluded. Most of these studies had few cases and short follow-up periods. ${ }^{5,6}$

These factors influence the reliability of PSA and raise the probability of false positives in obese patients, raising questions about the usefulness of PSA screening. It is necessary to study obesity and other confounding factors for PSA level at this time as Koreans become aged and the obesity population increases. Therefore, we studied the relationships between obesity indicators (weight, height, BMI, and WC), fasting blood sugar (FBS), lipid profiles, estimated glomerular filtration rate (GFR), and PSA in Korean men undergoing prostate health check-up

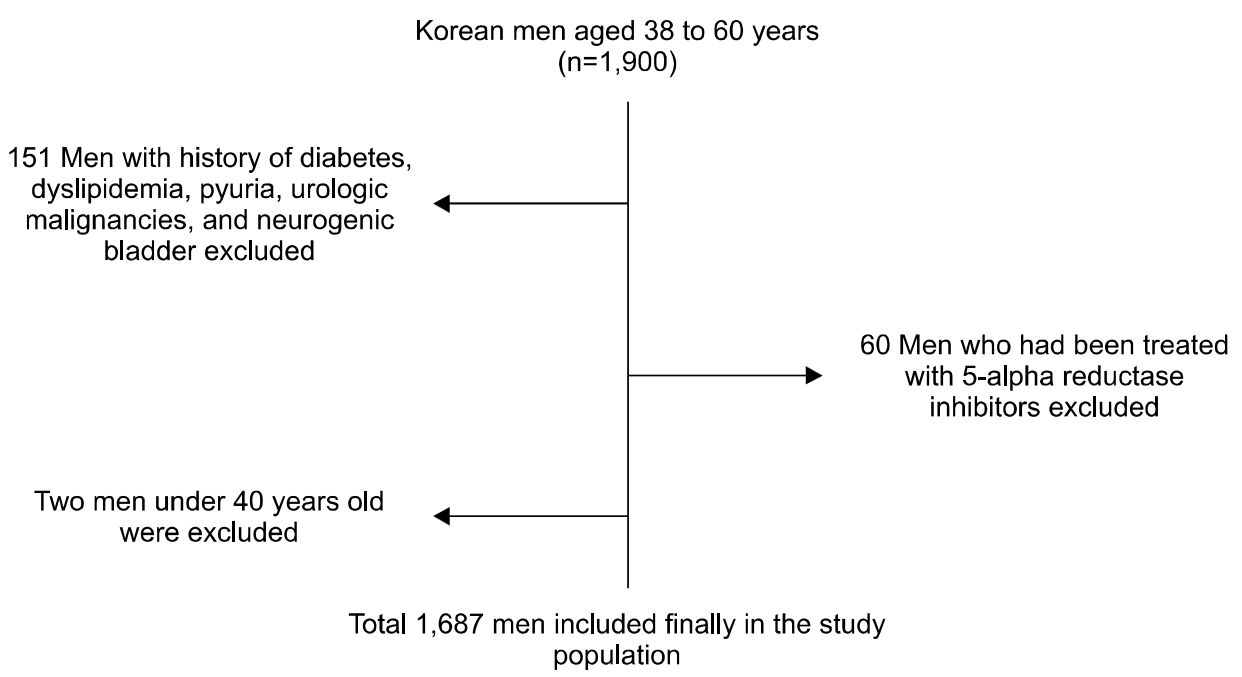

Fig. 1. Flow chart of study patient selection. including routine physical screening, transrectal ultrasonography, and digital rectal examination (DRE). The aim of this study was to determine whether obesity indices and confounding factors have effects on PSA.

\section{MATERIALS AND METHODS}

\section{Study population}

In this study, 1,900 Korean men aged 40 to 60 years undergoing prostate evaluation including transrectal ultrasonography as part of their health screening in our institution from February 2013 to September 2013 were included. This research was approved by the Ethics Committee of National Police Hospital (No. 11100176-201608-HR-005), and was performed in accordance with the Institutional Ethics Committee requirements. Because this study was a retrospective study, informed consent to individual subjects could be omitted. Men under 40 years old and with history of diabetes and dyslipidemia were excluded. Men who has been diagnosed with urologic diseases, including urogenital malignancies, and neurogenic bladder dysfunction or men who had taken 5-alpha reductase inhibitors were excluded. In addition, men with pyuria or bacteriuria were also excluded. A total 1,687 men were included finally in the study (Fig. 1).

\section{Assessment of obesity and prostate factors}

Weight, height, and WC of population were measured at the hospital by a nurse during the medical examination. After subjects stood relaxed, with arms folded comfortably, WC was
Men who had been treated
with 5-alpha reductase

inhibitors excluded 
measured at the level of uppermost border of hipbones (iliac crest). A series of 2 measurements were taken by trained nurses. Then, the average of the 2 measured values was obtained. BMI was calculated as weight in kilograms divided by squared height in meters $\left(\mathrm{kg} / \mathrm{m}^{2}\right)$.

All men were requested to complete a screening examination including FBS, lipid profiles, estimated GFR, PSA, and routine urinalysis. Blood specimens were sampled after minimum 8 hours fast from a vein. PSA analyses were completed using the test of automated chemiluminescent microparticle immunoassay analyzer Architect i2000 (Abbott Laboratories, Slingo, Ireland). FBS analyses were used the glucose oxidase method by DiaSys Diagnostic Systems GmbH (Holzheim, Germany). The estimated GFR was calculated according to the abbreviated 4-variable Modification of Diet in Renal Disease equation: $\mathrm{GFR}=186.3 \times($ serum creatinine $) \quad-1.154 \times($ age $)-0.203 .^{7} \quad$ All measurements were performed in a central laboratory according to the manufacturer's instructions. DRE and transrectal ultrasonography were carried out by a urologist. The total prostate volume (TPV) was checked using transrectal ultrasonography (UltraView 800, BKmedical, Herlev, Denmark). TPV was calculated assuming a prolate ellipsoid shape using the formula, prostate volume $=([\pi / 6] \times[$ anteroposterior $\times$ transverse $\times$ longitudin al length]).

\section{Statistical analysis}

Statistical analyses were conducted with PASW Statistics 18.0 (IBM SPSS, New York, NY, USA). Data were reported as means \pm standard deviation for continuous variables. All data were analyzed to evaluate a simple relationship of PSA level with age, TPV, height, weight, BMI, WC, FBS, lipid profiles, and estimated GFR by the Spearman correlation tests. Then, we evaluated the relationship of obesity indices with PSA after adjusting for age and TPV using the multiple linear regression test. The results were considered to be statistically significant when $\mathrm{p}$-value $<0.05$.

\section{RESULTS}

\section{Patient demographics}

The baseline characteristics in this study population are illustrated in Table 1. The mean age was $52.0 \pm 4.7$ years, and the mean PSA was $0.97 \pm 0.99 \mathrm{ng} / \mathrm{mL}$. The mean weight, BMI, and WC were $74.1 \pm 8.0 \mathrm{~kg}, 24.9 \pm 2.4 \mathrm{~kg} / \mathrm{m}^{2}$, and $88.5 \pm 6.3 \mathrm{~cm}$, re- spectively.

Relationship of PSA level with obesity indices, glucose, lipid, and renal function profiles tatistical analysis (Table 2) confirmed that age showed significant positive correlations with PSA $(r=0.108, p<0.01)$, TPV $(r=0.214, p<0.01)$, WC $(r=$ $0.113, \mathrm{p}<0.01)$, and FBS $(\mathrm{r}=0.075, \mathrm{p}<0.05)$ and significant negative correlations with height $(\mathrm{r}=-0.215, \mathrm{p}<0.01)$, weight $(\mathrm{r}=-0.115, \mathrm{p}<0.01)$, total cholesterol $(\mathrm{r}=-0.055, \mathrm{p}<0.05)$, lowdensity lipoprotein (LDL) cholesterol $(\mathrm{r}=-0.102, \mathrm{p}<0.01)$, triglyceride $(\mathrm{r}=-0.090, \mathrm{p}<0.01)$, and GFR $(\mathrm{r}=-0.213, \mathrm{p}<0.01)$.

PSA also demonstrated significant positive correlations with TPV ( $\mathrm{r}=0.349 ; \mathrm{p}<0.01)$ and LDL cholesterol $(\mathrm{r}=0.056, \mathrm{p}<$ $0.05)$ and significant negative correlations with height $(\mathrm{r}=-0.052, \mathrm{p}<$ 0.05), weight $(\mathrm{r}=-0.061, \mathrm{p}<0.05)$, and GFR $(\mathrm{r}=-0.096, \mathrm{p}<$ 0.01). After adjusting for age and TPV (Table 3), all obesity indices (weight, BMI, and WC) demonstrated negative correlations with PSA (beta $=-0.013, \mathrm{p}<0.001$; beta $=-0.039, \mathrm{p}<0.001$; and beta $=-0.010, \mathrm{p}=0.005$, respectively).

\section{DISCUSSION}

The incidence of PCa has increased not only in Korea but also globally, which is related to the aging of the population. Diabetes, obesity and dyslipidemia are some of the adult dis-

Table 1. Patients' characteristics $(n=1,687)$

\begin{tabular}{lc}
\hline \multicolumn{1}{c}{ Variable } & Mean \pm SD \\
\hline Age $(\mathrm{yr})$ & $52.0 \pm 4.7$ \\
PSA $(\mathrm{ng} / \mathrm{mL})$ & $0.97 \pm 0.99$ \\
TPV $\left(\mathrm{cm}^{3}\right)$ & $24.2 \pm 6.9$ \\
Height $(\mathrm{cm})$ & $172.6 \pm 4.3$ \\
Weight $(\mathrm{kg})$ & $74.1 \pm 8.0$ \\
BMI $\left(\mathrm{kg} / \mathrm{m}^{2}\right)$ & $24.9 \pm 2.4$ \\
WC $(\mathrm{cm})$ & $88.5 \pm 6.3$ \\
FBS $(\mathrm{mg} / \mathrm{dL})$ & $102.6 \pm 15.8$ \\
Total Chol $(\mathrm{mg} / \mathrm{dL})$ & $184.0 \pm 32.2$ \\
HDL Chol $(\mathrm{mg} / \mathrm{dL})$ & $42.4 \pm 9.9$ \\
LDL Chol $(\mathrm{mg} / \mathrm{dL})$ & $107.7 \pm 28.0$ \\
TG $(\mathrm{mg} / \mathrm{dL})$ & $167.6 \pm 119.0$ \\
CKD-EPI GFR $\left(\mathrm{mL} / \mathrm{min} / 1.73 \mathrm{~m}^{2}\right)$ & $98.2 \pm 15.5$ \\
\hline
\end{tabular}

SD: standard deviation, PSA: prostate-specific antigen, TPV: total prostate volume, BMI: body mass index, WC: waist circumference, FBS: fasting blood sugar, Chol: cholesterol, HDL: high-density lipoprotein, LDL: low-density lipoprotein, TG: triglyceride, CKD-EPI: Chronic Kidney Disease Epidemiology Collaboration, GFR: glomerular filtration rate. 


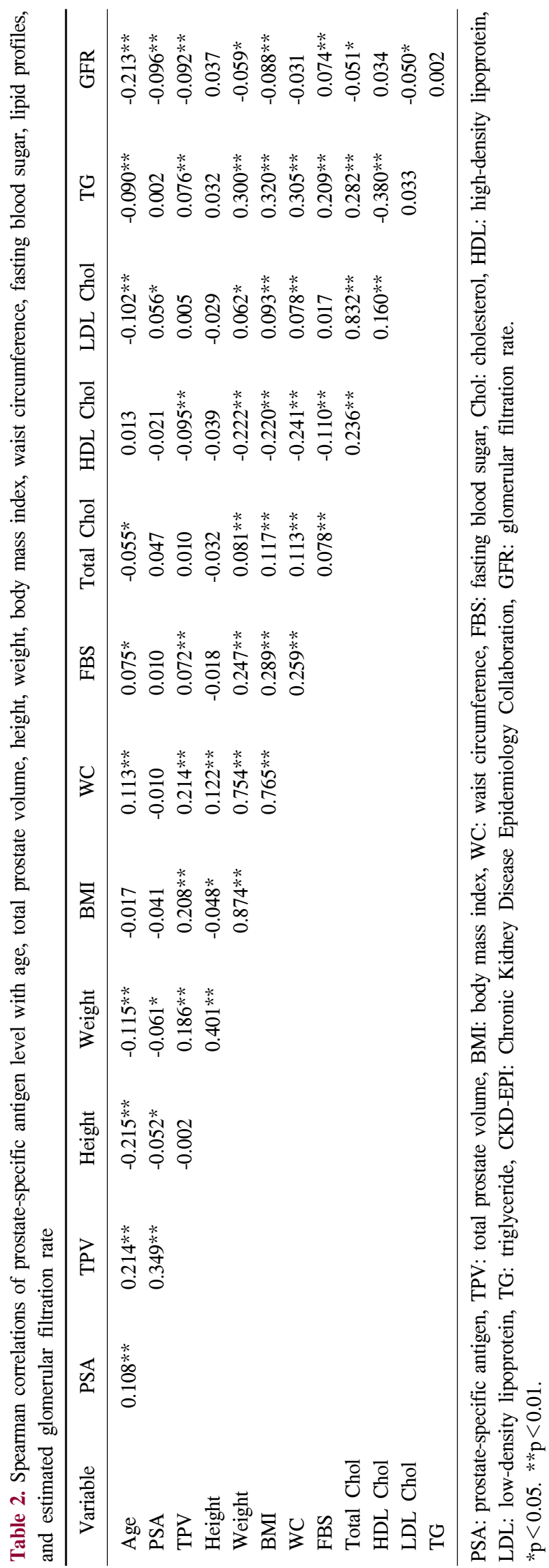

Table 3. Multiple linear regression test of obesity indices with prostate-specific antigen level

\begin{tabular}{cccr}
\hline & \multicolumn{3}{c}{ PSA } \\
\cline { 2 - 4 } Variable & Beta & $\begin{array}{c}\text { Standard } \\
\text { error }\end{array}$ & p-value \\
\hline Weight & & & \\
Model 1 & -0.006 & 0.003 & 0.040 \\
Model 2 & -0.005 & 0.003 & 0.128 \\
Model 3 & -0.013 & 0.003 & $<0.001$ \\
Body mass index & & & \\
Model 1 & -0.015 & 0.010 & 0.137 \\
Model 2 & -0.014 & 0.010 & 0.167 \\
Model 3 & -0.039 & 0.010 & $<0.001$ \\
Waist circumference & & & \\
Model 1 & -0.001 & 0.004 & 0.997 \\
Model 2 & -0.002 & 0.004 & 0.662 \\
Model 3 & -0.010 & 0.004 & 0.005 \\
\hline
\end{tabular}

PSA: prostate-specific antigen, model 1: not-adjusted, model 2: adjusting for age, model 3: adjusting for age and total prostate volume.

eases caused by the aging of the population. At present, It is unclear that what role they have on the development of PCa or PSA levels, and many scholars have a lot of questions about them.

This study showed that PSA level was negatively correlated with various indicators related to obesity after correction for the commonly known compounding factors age and prostate size. Since the 2000s, in many epidemiological studies, obesity has been suspected to be associated with the risk of various types of cancer as well as PCa. ${ }^{8}$ In several meta-analyses, obesity has been found to be associated with the risk of PCa. The relative risks reported in these studies was not constant, but increased with increasing BMI, from 1.01 (95\% confidence interval [CI], 1.00-1.02) per $1 \mathrm{~kg} / \mathrm{m}^{2}$ increment in BMI to 1.05 (95\% CI, 1.01 $-1.08) .{ }^{9}$ However, the results of each of the studies in the meta-analysis show variable results. In some studies, $\mathrm{PCa}$ was not associated with obesity. ${ }^{10}$ In few studies, obesity was considered a risk factor for $\mathrm{PCa},{ }^{11}$ and in others, it was a protective factor. ${ }^{12}$

One interesting point is that the results of the study differed according to geographical locations. In North American, obesity was not related to the risk of PCa, and on the other hand, in European and Australian studies, obesity generally increased the risk of PCa. ${ }^{8}$ It is suspected that these regional differences are often due to the different national PSA screening rates. 
Unlike the United States, European countries tend not to routinely perform PSA screening.

As shown in our study, obesity tends to lower PSA levels. Furthermore, in our study, weight, BMI and WC were negatively associated with serum PSA level independent of age and TPV. Although the relationship between PSA and PCa risk is currently unclear, many previous studies have shown negative correlations between PSA and various obesity-related indicators. In large studies in the United States, BMI was negatively correlated with PSA level after adjusting for age and race. ${ }^{13}$ In addition, in studies conducted in Asia, BMI reported negative correlations with PSA level. Unlike previous studies, we analyzed many confounding factors that may affect PSA levels including TPV, FBS, and dyslipidemia. Furthermore, obesity-related indicators included 3 different indicators such as body weight, BMI, and WC.

The effect of obesity on PSA is often explained by mechanism of hemodilution. It means that obesity results in a decrease in the PSA level in the blood as plasma volume increases in the human body. This theory suggested that the concentration of PSA is due to the function of plasma volume as well as the role of PSA poured into the circulation. ${ }^{14}$ There are other theories about PSA levels. It is so-called "steroid hormone metabolism hypothesis" that PSA levels are regulated by the concentration of steroid hormones. ${ }^{14}$ According to this theory, obesity affects PSA levels through various pathways and can affect the levels of human hormones and growth factors such as estrogen, testosterone, leptin, insulin, and insulin-like growth factor- 1 influencing prostate growth. ${ }^{15}$ In obese men, the amount of adipose tissue is high, which can increase the activity of aromatase by raising cyclic estrogen levels. ${ }^{15}$

Several measures of obesity (BMI, WC, weight, and body fat percentage) are usually correlated, but represent different forms of obesity. BMI is the most common measure of obesity, and WC is usually a measure of abdominal obesity. The staging reversal of $\mathrm{PCa}$ in obese people is explained by several hypotheses. It is somewhat more difficult to palpate the prostate gland in obese people clinically. In obese people, the blood PSA levels are lower for a variety of reasons and until now, there is lesser chance of having a prostate biopsy. ${ }^{16}$ In addition, it has been reported that obesity may promote PCa growth. In preclinical studies conducted to date, it is suspected that androgens promote PCa cell growth through the pathway of lipogenic gene expression, which causes lipid accumulation and regulates androgen signaling and lipogenesis. ${ }^{17}$ Castration-resistant PCa appears to produce androgen de novo from cholesterol and progesterone in the cancer cell, stimulating androgen receptors and growing PCa cells regardless of the concentration of circulating androgens. ${ }^{18}$ In experimental studies, fatty acid synthase polymorphisms are associated with the carcinogenesis of $\mathrm{PCa}$, and its high expression appears to be related to the development of high-risk PCa and high mortality in obese patients. ${ }^{19}$ Many articles mentioned the relationship of PCa, obesity, and various other components of the metabolic syndrome. Recently, a unique aggressive subtype of PCa, called TMPRSS2:ERG fusion has been introduced in relation to the effect of obesity on PCa prognosis. ${ }^{20}$

Whether diabetes itself has a protective effect against PCa or reduces PSA levels remains unclear issues. In our study, PSA level was not associated with FBS. However, in many studies, diabetes and the risk of PCa has been reported to be inversely correlated. A common hypothesis is that hyperinsulinemia lowers testosterone levels, which may lower the risk of $\mathrm{PCa} .{ }^{21}$ Unfortunately, the results of several studies were not consistent, and usually reported high prevalence of high-risk $\mathrm{PCa}$ in diabetic men. ${ }^{22}$ Although the mechanism is somewhat lacking in understanding, the theory that insufficient testosterone is involved in the proliferation cycle of the prostate, induces abnormal epithelial differentiation, and changes cancer cells into more aggressive cells is considered worthwhile. ${ }^{23}$

Some previous prospective studies have addressed the association between total cholesterol and high-risk $\mathrm{PCa}^{24}$ However, total cholesterol and PSA were not related in our study. Taken together, previous results suggested that total cholesterol have no significant effect on PSA levels. In recent large-scale study, triglycerides appeared to have an effect on the aggressiveness and severity of $\mathrm{PCa}^{25}$ In addition, in the in vitro studies, triglyceride-rich remnant lipoproteins have been promoted carcinogenesis according to up-regulating cellular signaling, such as MEK/ERK and Akt pathways, involved in modulating cell proliferation and growth, apoptosis, cell cycle arrest, and lipid biosynthesis. ${ }^{26}$ One large study in China reported that triglycerides were inversely correlated to PSA levels. ${ }^{27}$ However, this study did not find a relationship between triglyceride and PSA, and it seems that LDL cholesterol is weakly related to PSA. Therefore, further studies for relationship between lipid and PSA should be studied in patients with PCa because most of the current research has been done from healthy population. 
This is a relatively large study to investigate the relationship between obesity, glucose and lipid and PSA. Our study population consisted of relatively healthy middle-aged men and included only those participants who did not have a history of diabetes and dyslipidemia prior to participation. In addition, our study was based on prediagnostic serologically measured values consistently in a single laboratory. This can help prevent reverse causation between causes and effects. However, our study was based solely on single baseline laboratory values and could not reflect sequential effects of metabolic components to PSA level over long periods of time. If repeated measurements are made throughout the life cycle, these limitations can be overcome in the future. Our study could not investigate lifestyle-related factors such as smoking history or dietary habit. However, according to the literature known to date, the aforementioned lifestyle-related factors are not considered to be associated with PSA levels.

In conclusion, this study found that several indices associated with obesity had an effect on PSA levels in Korean middle-aged men aged 38 to 60 years who underwent routine health examination, and these factors were independent of age and TPV. In the future, it may be necessary to provide reference values considering the effects of obesity indices for middle-aged men who perform PSA screening for the first time in life, unlike men aged 60 years or older.

\section{CONFLICT OF INTEREST}

The authors claim no conflicts of interest.

\section{REFERENCES}

1. Jung KW, Won YJ, Oh CM, Kong HJ, Cho H, Lee JK, et al. Prediction of cancer incidence and mortality in Korea, 2016. Cancer Res Treat 2016;48:451-7.

2. Pischon T, Boeing H, Weikert S, Allen N, Key T, Johnsen $\mathrm{NF}$, et al. Body size and risk of prostate cancer in the European prospective investigation into cancer and nutrition. Cancer Epidemiol Biomarkers Prev 2008;17:3252-61.

3. Bassett JK, Severi G, Baglietto L, MacInnis RJ, Hoang HN, Hopper JL, et al. Weight change and prostate cancer incidence and mortality. Int J Cancer 2012;131:1711-9.

4. Naito M, Asai Y, Mori A, Fukada Y, Kuwabara M, Katase $\mathrm{S}$, et al. Association of obesity and diabetes with serum prostate-specific antigen levels in Japanese males. Nagoya J Med Sci 2012;74:285-92.
5. Pelucchi C, Serraino D, Negri E, Montella M, Dellanoce C, Talamini R, et al. The metabolic syndrome and risk of prostate cancer in Italy. Ann Epidemiol 2011;21:835-41.

6. Häggström C, Stocks T, Ulmert D, Bjørge T, Ulmer H, Hallmans G, et al. Prospective study on metabolic factors and risk of prostate cancer. Cancer 2012;118:6199-206.

7. Levey AS, Stevens LA, Schmid CH, Zhang YL, Castro AF 3rd, Feldman HI, et al. A new equation to estimate glomerular filtration rate. Ann Intern Med 2009;150:604-12.

8. Renehan AG, Tyson M, Egger M, Heller RF, Zwahlen M. Body-mass index and incidence of cancer: a systematic review and meta-analysis of prospective observational studies. Lancet 2008;371:569-78.

9. MacInnis RJ, English DR. Body size and composition and prostate cancer risk: systematic review and meta-regression analysis. Cancer Causes Control 2006;17:989-1003.

10. Baillargeon J, Platz EA, Rose DP, Pollock BH, Ankerst DP, Haffner S, et al. Obesity, adipokines, and prostate cancer in a prospective population-based study. Cancer Epidemiol Biomarkers Prev 2006;15:1331-5.

11. Engeland A, Tretli S, Bjørge T. Height, body mass index, and prostate cancer: a follow-up of 950000 Norwegian men. $\mathrm{Br}$ J Cancer 2003;89:1237-42.

12. Wright ME, Chang SC, Schatzkin A, Albanes D, Kipnis V, Mouw T, et al. Prospective study of adiposity and weight change in relation to prostate cancer incidence and mortality. Cancer 2007;109:675-84.

13. Baillargeon J, Pollock BH, Kristal AR, Bradshaw P, Hernandez J, Basler J, et al. The association of body mass index and prostate-specific antigen in a population-based study. Cancer 2005; 103:1092-5.

14. Fowke JH, Matthews CE. PSA and body composition by dual X-ray absorptiometry (DXA) in NHANES. Prostate 2010;70: 120-5.

15. Skolarus TA, Wolin KY, Grubb RL 3rd. The effect of body mass index on PSA levels and the development, screening and treatment of prostate cancer. Nat Clin Pract Urol 2007;4:60514.

16. Allott EH, Masko EM, Freedland SJ. Obesity and prostate cancer: weighing the evidence. Eur Urol 2013;63:800-9.

17. Freeman BC, Yamamoto KR. Disassembly of transcriptional regulatory complexes by molecular chaperones. Science 2002; 296:2232-5.

18. Locke JA, Guns ES, Lubik AA, Adomat HH, Hendy SC, Wood CA, et al. Androgen levels increase by intratumoral de novo steroidogenesis during progression of castration-resistant prostate cancer. Cancer Res 2008;68:6407-15.

19. Flavin R, Zadra G, Loda M. Metabolic alterations and targeted therapies in prostate cancer. J Pathol 2011;223:283-94.

20. Pettersson A, Lis RT, Meisner A, Flavin R, Stack EC, Fiorentino $\mathrm{M}$, et al. Modification of the association between obesity and lethal prostate cancer by TMPRSS2:ERG. J Natl 
Cancer Inst 2013;105:1881-90.

21. Tsilidis KK, Kasimis JC, Lopez DS, Ntzani EE, Ioannidis JP. Type 2 diabetes and cancer: umbrella review of meta-analyses of observational studies. BMJ 2015;350:g7607.

22. Moreira DM, Anderson T, Gerber L, Thomas JA, Bañez LL, McKeever MG, et al. The association of diabetes mellitus and high-grade prostate cancer in a multiethnic biopsy series. Cancer Causes Control 2011;22:977-83.

23. García-Cruz E, Piqueras M, Huguet J, Peri L, Izquierdo L, Musquera M, et al. Low testosterone levels are related to poor prognosis factors in men with prostate cancer prior to treatment. BJU Int 2012;110(11 Pt B):E541-6.

24. Morote J, Celma A, Planas J, Placer J, de Torres I, Olivan $\mathrm{M}$, et al. Role of serum cholesterol and statin use in the risk of prostate cancer detection and tumor aggressiveness. Int $\mathbf{J}$ Mol Sci 2014;15:13615-23.

25. Arthur R, Møller H, Garmo H, Holmberg L, Stattin P, Malmstrom H, et al. Association between baseline serum glucose, triglycerides and total cholesterol, and prostate cancer risk categories. Cancer Med 2016;5:1307-18.

26. Sekine Y, Koike H, Nakano T, Nakajima K, Takahashi S, Suzuki K. Remnant lipoproteins induced proliferation of human prostate cancer cell, PC-3 but not LNCaP, via low density lipoprotein receptor. Cancer Epidemiol 2009;33:16-23.

27. Liu M, Wang JY, Zhu L, Wan G. Body mass index and serum lipid profile influence serum prostate-specific antigen in Chinese men younger than 50 years of age. Asian J Androl 2011;13:640-3. 